\title{
Effect of Fecal Microbiota Transplantation on Primary Hypertension and the Underlying Mechanism of Gut Microbiome Restoration: Protocol of a Randomized, Double-Blinded, Placebo- Controlled Study
}

\section{Luyun Fan}

Chinese Academy of Medical Sciences \& Peking Union Medical College Fuwai Hospital

Jie Ren

Shanxi Bethune Hospital

\section{Youren Chen}

The Second Affiliated Hospital of Shantou University

\section{Yang Wang}

Fuwai Hospital Chinese Academy of Medical Sciences

\section{Zihong Guo}

Fuwai Yunnan Cardiovascular Hospital

\section{Peili Bu}

Qilu Hospital of Shandong University

\section{Jinfeng Yang}

The People's Hospital of Ji Xian District

\section{Wenjun Ma}

Chinese Academy of Medical Sciences \& Peking Union Medical College Fuwai Hospital

\section{Bingpo Zhu}

Fuwai Hospital, Chinese Academy of Medical Sciences and Peking Union Medical College

\section{Yanyan Zhao}

Fuwai Hospital Chinese Academy of Medical Sciences

Jun Cai ( $\sim$ caijun7879@126.com )

Chinese Academy of Medical Sciences \& Peking Union Medical College Fuwai Hospital

\section{Study protocol}

Keywords: Hypertension, Blood Pressure, Fecal Microbiota Transplantation, Therapy, Randomized Controlled Trial, Microbiome, Metabolome 
Posted Date: August 2nd, 2021

DOI: https://doi.org/10.21203/rs.3.rs-706375/v1

License: (c) (i) This work is licensed under a Creative Commons Attribution 4.0 International License. Read Full License

Version of Record: A version of this preprint was published at Trials on February 24th, 2022. See the published version at https://doi.org/10.1186/s13063-022-06086-2. 


\section{Title}

Effect of Fecal Microbiota Transplantation on Primary Hypertension and the Underlying Mechanism of Gut Microbiome Restoration: Protocol of a Randomized, Double-Blinded, PlaceboControlled Study

\section{Names protocol contributors}

Luyun Fan', M.D., Ph.D., Jie Ren ${ }^{2}$, M.D., Youren $C^{3}{ }^{3}$, M.D., Yang Wang ${ }^{4}$, Zihong Guo ${ }^{5}$, M.D., Peili Bu${ }^{6}$, M.D., Ph.D., Jinfeng Yang ${ }^{7}$, M.D., Wenjun Ma'1 , M.D., Ph.D., Bingpo Zhu ${ }^{8}$, M.D., Yanyan Zhao $^{4}$, Jun Cai, M.D., Ph.D. ${ }^{1}$

\section{Abstract}

- Background: Hypertension is in current the leading modifiable cause of global morbidity and mortality, contributing to substantial health and financial burdens. Although multiple explorations on management models and innovative therapeutic strategies of hypertension, vacancy still occur in the field with poor control rate reflected and lacking of novel, effectively clinical-translated medication or intervention options. Recent animal and human studies repeatedly confirmed a link between microbiota and hypertension. Of note is our previous study establishing a cause-and-effect relationship between gut microbiota and blood pressure elevation. A hypothesis of gut microbiota intervention on treating hypertension is thus postulated with fecal microbiota transplantation(FMT) from healthy donors performed.

- Methods: A multi-center, central randomized, placebo-controlled, double-blinded clinical trial is performed in 120 grade 1 hypertensive patients for overall three months. All recruited patients will be randomly assigned in a 1:1 ratio into orally-taken FMT capsules or placebo capsules with three interventions on day 1 , day 7 and day 14 in separate, and followed up on day 30 , day 60 and day 90 . The primary outcome is the change for office systolic blood pressure from baseline to day 30 follow-up. Main secondary outcomes are BP indicators including changes in systolic and diastolic blood pressure from office, home, and 24-hour ambulatory blood pressure monitoring, assessments of ankle-branchial index and pulse wave velocity, profiling of fecal microbial composition and function, profiling of fecal and serum metabolome, changes in levels of blood glucose, blood lipids and body mass index, assessment of adverse events as a measure of safety.

- Discussion: Stretching from our previous research on the role of gut microbiota in the 
pathogenesis of hypertension, this study serves as a clinical translation advancement and firstly explores the potential of fecal microbiota transplantation on treating hypertension. Underlying mechanisms particularly on anchoring specific microorganisms or their postbiotics contributing to blood pressure amelioration will also be investigated via multiple approaches such as metagenomic sequencing and metabolomic profiling.

- Trial registration: ClinicalTrials.gov Identifier: NCT04406129, registried on May $28^{\text {th }}, 2020$, https://clinicaltrials.gov/ct2/show/NCT04406129

\section{Keywords}

Hypertension, Blood Pressure, Fecal Microbiota Transplantation, Therapy, Randomized Controlled Trial, Microbiome, Metabolome

\section{Administrative information}

Note: the numbers in curly brackets in this protocol refer to SPIRIT checklist item numbers. The order of the items has been modified to group similar items (see http://www.equator-network.org/reportingguidelines/spirit-2013-statement-defining-standard-protocol-items-for-clinical-trials/).

\begin{tabular}{|l|l|}
\hline Title $\{1\}$ & $\begin{array}{l}\text { Effect of Fecal Microbiota Transplantation on Primary } \\
\text { Hypertension and the Underlying Mechanism of Gut } \\
\text { Microbiome Restoration: Protocol of a Randomized, Double- } \\
\text { Blinded, Placebo-Controlled Study }\end{array}$ \\
\hline Trial registration $\{2 \mathrm{a}$ and $2 \mathrm{~b}\}$. & $\begin{array}{l}\text { ClinicalTrials.gov Identifier: NCT04406129, registried on May } \\
\text { 28th, 2020, https://clinicaltrials.gov/ct2/show/NCT04406129 }\end{array}$ \\
\hline Protocol version $\{3\}$ & $\begin{array}{l}\text { Version 3.3, dated 26 February 2021 } \\
\text { Funding }\{4\}\end{array}$ \\
\hline $\begin{array}{l}\text { This work is funded by grants from the National Natural } \\
\text { Science Foundation of China (Project ID. 81630014) and from } \\
\text { Beijing Outstanding Young Scientist Program (Project ID, } \\
\text { BJJWZYJH01201910023029). }\end{array}$ \\
\hline
\end{tabular}


1. Luyun Fan, M.D., Ph.D., Hypertension Center, State Key Laboratory of Cardiovascular Disease, National Center for Cardiovascular Diseases, Fuwai Hospital, Chinese Academy of Medical Sciences and Peking Union Medical College, Beijing, China.

2. Jie Ren, M.D., Shanxi Bethune Hospital, Taiyuan, Shanxi, China.

3. Youren Chen, M.D., The Second Affiliated Hospital of Shantou University, Shantou, Guangdong, China.

4. Yang Wang, Medical Research \& Biometrics Center, National Center for Cardiovascular Diseases, Fuwai Hospital Chinese Academy of Medical Sciences, Beijing, China.

5. Zihong Guo, M.D., Fuwai Yunnan Cardiovascular Hospital, Kunming, Yunnan, China.

6. Peili Bu, M.D., Ph.D., Qilu Hospital of Shandong University, Jinan, Shandong, China.

7. Jinfeng Yang, M.D., The People's Hospital of Ji Xian District, Tianjin, China.

8. Wenjun Ma, M.D., Ph.D., Hypertension Center, State Key Laboratory of Cardiovascular Disease, National Center for Cardiovascular Diseases, Fuwai Hospital, Chinese Academy of Medical Sciences and Peking Union Medical College, Beijing, China.

9. Bingpo Zhu, M.D., Southern University of Science and Technology Hospital, Shenzhen, China.

10. Yanyan Zhao, Medical Research \& Biometrics Center, National Center for Cardiovascular Diseases, Fuwai Hospital Chinese Academy of Medical Sciences, Beijing, China.

11. Jun Cai, M.D., Ph.D., Hypertension Center, State Key Laboratory of Cardiovascular Disease, National Center for Cardiovascular Diseases, Fuwai Hospital, Chinese Academy of Medical Sciences and Peking Union Medical College, Beijing, China. 


\begin{tabular}{|l|l|}
\hline $\begin{array}{l}\text { Name and contact information for } \\
\text { the trial sponsor }\{5 \mathrm{~b}\}\end{array}$ & $\begin{array}{l}\text { Prof. Jun Cai (Principal Investigator), State Key Laboratory of } \\
\text { Cardiovascular Disease, National Center for Cardiovascular } \\
\text { Diseases, Fuwai Hospital, Chinese Academy of Medical } \\
\text { Sciences and Peking Union Medical College; } \\
167 \text { Beilishi Road, Xichen District, 100037, Beijing, China; } \\
\text { E-mail: caijun7879@126.com; caijun@fuwaihospital.org; } \\
\text { Phone: 01088322161 }\end{array}$ \\
\hline \multirow{2}{*}{$\begin{array}{l}\text { The of sponsor }\{5 \mathrm{c}\} \\
\text { procured funding. The study funders will not involve the study } \\
\text { design, implementation, data interpretation, and result } \\
\text { publication. }\end{array}$} & \\
&
\end{tabular}

\section{Introduction}

\section{Background and rationale $\{6 \mathrm{a}\}$}

Cardiovascular Diseases (CVDs) are currently the leading cause of global health loss, accounting for one-third of deaths worldwide(1) and estimated to cause 7.8 million premature deaths in 2025 (2). Primary hypertension is a most prevalent cardiovascular disease affecting $\sim 30 \%$ adult population worldwide, and becomes a severe public health issue because of low control rate, high morbidity and leading to $\sim 19 \%$ global deaths $(3,4)$. Despite explorations on pharmacological and devicebased therapies over 60 years, a few treatment options are currently provided which stress advances of novel hypotheses on this field.

Albeit regulated by multifactorial genetic and environmental factors, burgeoning studies indicated the role of microbiota in blood pressure(BP) homeostasis (5). In germ-free(GF) animal models, angiotensin II(Ang II)-induced hypertension cannot be replicated (6). Decreased diversity and discordant composition of gut microbiota (from healthy controls) presented in several hypertensive cohorts (7-10). Fecal microbiota transplantation from multiple hypertensive animal models can increase BP in controls (11). Our previous study firstly confirmed that transplants from hypertensive patient feces reshaped the gut microbiota of healthy GF mice and rendered significant BP elevation (9). Of note, modulating intestinal flora via transplantation from healthy rats could dramatically reduce $B P$ in high salt-induced hypertensive animals(12). Oral antibiotics such as minocycline and vancomycin can assist on treatment of hypertension (13). Supplementary probiotics also modestly ameliorated high BP in rodents and/or humans (14-17). A hypothesis is indeed raised that gut microbiota intervention might be a potential approach to ameliorate hypertension.

Fecal microbiota transplantation (FMT) is a strategy for entire gut microbiome transplantation from healthy donors to the diseased recipient. Since 2013, FMT is recommended for treatment on recurrent Clostridium difficile infection resistant to standard-of-care therapies (18). Potential therapeutic roles of FMT via affecting patients' gut microbiota (19) has been examined on other gastrointestinal diseases such as inflammatory bowel disease(IBD) $(20,21)$ and irritable bowel disease (22), and extraintestinal diseases such as metabolic syndrome $(23,24)$, obese and type 2 diabetes mellitus $(25,26)$. The intervention is generally with mild and mainly self-limiting safety 
concerns (27). Of note is no study of entire gut microbial ecology restoration on hypertensive patients therapy assessed and its durability after FMT intervention.

We herein develop a multicenter, randomized, placebo-controlled, blinded study utilizing FMT intervention to explore the efficacy of gut microbiota intervention on essential hypertension. We postulate that FMT intervention presents a superior BP-lowering effect compared with placebo. Secondary outcomes also include changes in recipient fecal microbial composition and function, fecal and serum metabolome, fasting blood glucose and lipid levels, ankle branchial index(ABI), body mass index(BMI), and occurrence of adverse events as a measure of safety outcomes. The study also presents as the clinical translational section of the research project "The Role of Gut Microbiota in the Pathogenesis of Essential Hypertension" (Project ID 81630014, funded by National Natural Science Foundation of China).

\section{Objectives $\{7\}$}

This study primarily aims to assess whether FMT ameliorates elevated blood pressure in patients with grade 1 essential hypertension. Also, this study will determine whether any BP changes are associated with alteration in fecal microbiome, fecal and serum metabolome of hypertensive patients prior to and after FMT. Of note are the microbial structure and function alterations in recipient microbiota and their durability. Durability for clinical response after initial response to FMT will also be evaluated in participants with hypertension. Other indicators including fasting blood glucose and lipid levels, ABI, BMI, and occurrence of adverse events as a measure of safety outcomes will also be assessed.

\section{Trial design $\{8\}$}

This study is an investigator-initiated, multicenter, randomized, double-blinded, placebo-controlled clinical trial for overall three months.

After meeting the eligible criteria, 120 participants are randomly assigned into two arms, in which the experimental group will then be offered three separate "FMT capsules" treatment (Day 1, Day 7, Day 14 ) and the control group will then be offered three separate "Placebo capsules" therapy (Day 1, Day 7, Day 14). Except for the comprehensive examination before and at the end of the trial (Month 3), participants in both arms will be followed up at Day 7, Day 14, Day 30(Month 1), Day 60(Month 2), Day 90(Month 3) to observe the clinic BP and other clinical indicators, respectively. Adverse events and treatment for other accompanying diseases will also be recorded at each visit, as well as fecal and blood samples will be collected before the trial and at each visit for future metagenomic and metabolomic profiling analysis. All staff involved in clinical assessments and randomized participants will be blinded.

The protocol report refers to the SPIRIT reporting guidelines(28).

\section{Methods: Participants, interventions and outcomes}

\section{Study setting $\{9\}$}

This study is conducted by Fuwai Hospital, Chinese Academy of Medical Sciences, National Center for Cardiovascular Diseases, State Key Laboratory of Cardiovascular Diseases. Patients with grade 1 essential hypertension is recruited via the outpatient clinics at implementation centers including Fuwai Hospital, Chinese Academy of Medical Sciences (Beijing), Fuwai Yunnan Cardiovascular Hospital(Yunnan Province), Qilu Hospital of Shandong University(Shandong Province), the Second Affiliated Hospital of Shantou University Medical College(Guangdong Province), The People's 
Hospital of Ji Xian District(Tianjin), Shanxi Bethune Hospital(Shanxi Province), and South University of Science and Technology Hospital(Shenzhen).

\section{Eligibility criteria $\{10\}$}

\section{Inclusion Criteria:}

1. Age 18 60 years

2. Established diagnosis of grade 1 Hypertension (initial diagnosis or free from antihypertensive drugs within a month): $140 \mathrm{mmHg} \leq$ Office SBP $<160 \mathrm{mmHg}$ and/or $90 \leq$ Office $\mathrm{DBP}<100 \mathrm{mmHg}$ for three measurements at different days without any antihypertensive medications, according to the "2010 Chinese Guidelines for Prevention and Treatment of Hypertension" (29)

3. Participants with written informed consent after thorough explanation

\section{Exclusion Criteria:}

1. Antibiotics or probiotics usage within last month before the study

2. Participants of other clinical trials related to hypertension currently or within last 3 months

3. Antihypertensive medications usage currently or within last month

4. Diagnosis of secondary hypertension

5. Severe hepatic or renal diseases (over 3-times elevation of alanine transaminase, elevated serum creatinine over $2.5 \mathrm{mg} / \mathrm{dl}$ [221 $\mathrm{\mu mol} / \mathrm{L}]$, estimated glomerular filtration rate less than 30 $\mathrm{mL} / \mathrm{min} / 1.73 \mathrm{~m}^{2}$, or end-stage kidney dysfunction requiring dialysis)

6. History of stroke, including hemorrhagic stroke, large artery atherosclerotic cerebral infarction(LAACI), but not transient ischemic attack(TIA) or lacunar infarction

7. History of hospitalization within last six months caused by myocardial infarction; History for coronary revascularization including percutaneous transluminal coronary intervention $(\mathrm{PCl})$ or coronary artery bypass grafting (CABG) within last year; Anticipated PCl or CABG surgery in the following 12 months

8. Sustained atrial fibrillation or arrhythmias at recruitment disturbing the electronic BP measurement

9. NYHA class III-IV heart failure; Hospitalization for chronic heart failure exacerbation within last 6 months

10. Severe valvular diseases; Potential for surgery or percutaneous valve replacement within the study period

11. Dilated cardiomyopathy; Hypertrophic cardiomyopathy; Rheumatic cardiac disease; Congenital cardiac disease

12. Other severe diseases influencing the entry or survival of participants, such as malignant tumor or acquired immune deficiency syndrome

13. Cognitive impairment or severe neuropsychiatric comorbidities who are incapable of providing their own informed consent

14. Participants preparing for or under pregnancy and/or lactation

15. Other conditions inappropriate for recruitment according to the investigators.

\section{Who will take informed consent? \{26a\}}

Informed consent forms are prepared according to guidelines of and approved by the local ethical boards of all participated centers. The investigator or his/her designee will present the study to potential study participants and will answer study-related questions from the participants, such as study objectives, interventions, potential benefits, risks, and alternatives. The participants will read the information consent forms and have the opportunity to communicate with relatives prior to signing and dating the consent. Participation in this study is voluntary and entirely up to the participant's decision. Even if the participants do not participate, their medical services will not be affected in any way. If participants decide to participate, they also have the right to withdraw at any time and will not be influenced now or in the future on treatment. Once the participants decide to stop participating in the study, they will no longer be provided with the study interventions. 


\section{Additional consent provisions for collection and use of participant data and biological specimens $\{26 \mathrm{~b}\}$}

Information on clinical data and fecal and blood sample collection have already been included in the informed consent. The volume of fecal samples $(5 \mathrm{~g}$ per visit) and blood samples (10ml per visit) taken will not leads to side effects. The participation for each patient is confidential and their identity will be confined within the study centers under participants' agree.

\section{Interventions}

\section{Explanation for the choice of comparators $\{6 \mathbf{b}\}$}

Participants in the "placebo capsule" group will orally take placebo capsules on Day 1, Day 7 ( \pm 1d) and Day 14 ( $\pm 1 d)$. The placebo capsules are with identically appearing powder that do not contain donor stool or any active drug.

Intervention description $\{11 \mathrm{a}\}$

Participants in the "FMT capsule" group will orally take the FMT capsules on Day 1, Day 7 ( \pm 1 d) and Day 14 ( \pm 1d). The FMT capsules (China National Intellectual Property Administration, patent ID. CN 104922158 B) are prepared via processing extensively screened donor stool in reference to current consensus on FMT $(19,30-32)$, and stored at $-80^{\circ} \mathrm{C}$ freezers before recovery for use. Light, high liquid meals at the day before intervention and fasting for at least four hours prior to and two hours after intervention are required.

\section{Criteria for discontinuing or modifying allocated interventions $\{11 \mathrm{~b}\}$}

The discontinuation criteria include withdrawal of informed consent, loss to follow-up, occurrence of serious adverse events, complications, special physiological changes or other medical reasons precluding continuation for the study from investigators' assessment, significant deviation of the intervention algorithm, which probably affects outcome evaluation of the study.

\section{Strategies to improve adherence to interventions $\{11 \mathrm{c}\}$}

Before inclusion, the willingness for compliance is repeated confirmed among potential participants. An advantage of compliance is that all capsules are taken at three intervention visits within two weeks. The FMT capsules and placebo capsules will be stored, delivered and recovered by investigators and/or patient care providers from the implementation centers. All capsules are stored at $-80^{\circ} \mathrm{C}$ freezers and delivered from the trial responsible center into implementation centers via dry ice. Before intervention, the investigators and/or patient care providers will recover capsules within $37^{\circ} \mathrm{C}$ water bath and make detailed records of the number of distributed, administrated, and returned capsules. All recovered capsules at each visit will be orally taken within 30 minutes under clinical supervision. The remaining study capsules will be returned to the responsible center. Participant compliance rate will be evaluated defined as actual number of capsules used divided by anticipated number of capsules used $\times 100 \%$. Good compliance is defined as a compliance rate of $80 \%-120 \%$; poor compliance is defined as a compliance rate less than $80 \%$ or more than $120 \%$. If poor compliance identified, thorough communication between investigators and patients will be performed to reach possible solutions. In addition, alignment of gut microbial composition between FMT capsules and stool samples from recipients at different timepoints will be performed to assess the engraftment of microbiota, which will provide additional information for participant adherence in part. 


\section{Relevant concomitant care permitted or prohibited during the trial $\{11 \mathrm{~d}\}$}

In order to minimize the interference caused by dietary environmental factors on intestinal flora, recruited participants at each arm are required to record dietary history before each study visit, to make no dietary habit changes throughout the trial and avoid the use of probiotics, prebiotics, or antibiotics unless otherwise specific conditions recorded. During the treatment and follow-up period, participants should maintain a normal pattern of daily activities, such as physical activity, lifestyle, and habits. The conventional treatment for accompanying diseases in participants can remain unchanged and will be recorded. Treatment for adverse events are permitted and will be recorded.

\section{Provisions for post-trial care $\{30\}$}

In accordance with the inform consent, appropriate medical care and assistance will be provided rather than direct financial compensation for participants suffer harm relevant to trial participation. Also, close monitoring and management will be performed for those participants according to relevant laws and regulation.

\section{Outcomes $\{12\}$}

Our primary hypothesis is a BP-lowering effect of gut microbiota restoration via FMT compared to placebo in grade 1 hypertensive patients. Accordingly, the primary outcome is defined as change for office systolic blood pressure(SBP) from baseline to one-month follow-up (Day 30).

Other indicators related to BP evaluation, safety concerns and potential mechanism exploration will be assessed as main secondary outcomes, including:

1. The change in office systolic blood pressure(SBP).

2. The change in office diastolic blood pressure (DBP).

3. The change in average SBP and DBP during the 24-hour assessment, day, and night via 24-h ambulatory BP monitoring(ABPM).

4. The change in home SBP and DBP via home BP monitoring.

5. Target organ damage assessment from baseline to final visit, including: a) the change in anklebrachial blood pressure index $(\mathrm{ABI})$ as an objective measurement of arterial insufficiency based on the ratio of ankle systolic pressure to brachial systolic pressure; b) the change in pulse wave velocity as the distance divided by the time for a pulse delay between two arterial sites.

6. Number of participants with adverse events (AEs) as a measure of safety.

7. Changes in fecal microbiota composition and function pre- and post-intervention (FMT or placebo) via metagenomic analysis, stratified by: a) randomization; b) change in office SBP.

8. Durability of engraftment of donor microbiome following FMT, measured by similarity comparison of fecal microbiota composition between donor and recipient.

9. Changes in fecal metabolite composition and function pre- and post-intervention (FMT or Placebo) via metabolomic analysis, stratified by: a) randomization; b) change in office SBP.

10. Changes in blood metabolite composition and function pre- and post-intervention (FMT or Placebo) via metabolomic analysis, stratified by: a) randomization; b) change in office SBP.

11. The change in fasting blood glucose level from baseline to final visit.

12. The change in blood lipids level (total cholesterol, total triglyceride, low density lipoprotein cholesterol, high density lipoprotein cholesterol) from baseline to final visit.

13. The change in body mass index from baseline to final visit.

\section{Participant timeline $\{13\}$}

Table 1. The schedule of enrolment, interventions, and assessments. 


\begin{tabular}{|c|c|c|c|c|c|c|c|c|}
\hline & Enrollment & Allocation & \multicolumn{5}{|c|}{ Follow-up } & \multirow{2}{*}{$\begin{array}{c}\begin{array}{c}\text { Close- } \\
\text { out }\end{array} \\
\text { Day } 90 \\
( \pm 3 d)\end{array}$} \\
\hline Work Flow & -7 days & Day 0 & $\begin{array}{c}\text { Day } \\
1\end{array}$ & $\begin{array}{l}\text { Day } 7 \\
( \pm 1 d)\end{array}$ & $\begin{array}{l}\text { Day 14 } \\
( \pm 1 d)\end{array}$ & $\begin{array}{l}\text { Day 30 } \\
( \pm 3 d)\end{array}$ & $\begin{array}{l}\text { Day 60 } \\
( \pm 3 d)\end{array}$ & \\
\hline ENROLMENT: & & & & & & & & \\
\hline Eligibility screen & $\checkmark$ & & & & & & & \\
\hline Informed consent & $\checkmark$ & & & & & & & \\
\hline Allocation & & $\checkmark$ & & & & & & \\
\hline INTERVENTIONS: & & & & & & & & \\
\hline FMT capsules & & & $\checkmark$ & $\checkmark$ & $\checkmark$ & & & \\
\hline Placebo capsules & & & $\checkmark$ & $\checkmark$ & $\checkmark$ & & & \\
\hline ASSESSMENTS: & & & & & & & & \\
\hline $\begin{array}{r}\text { Comorbid Diseases \& } \\
\text { Treatment }\end{array}$ & $\checkmark$ & & & & & & & \\
\hline Physical Examination & $\checkmark$ & & & & & & & $\checkmark$ \\
\hline $\begin{array}{r}\text { Office BP and Heart } \\
\text { rate }\end{array}$ & $\checkmark$ & & & $\checkmark$ & $\checkmark$ & $\checkmark$ & $\checkmark$ & $\checkmark$ \\
\hline $24 h A B P M$ & $\checkmark$ & & & & & $\checkmark$ & & $\checkmark$ \\
\hline Blood routine & $\checkmark$ & & & & & & & $\checkmark$ \\
\hline Urine routine & $\checkmark$ & & & & & & & $\checkmark$ \\
\hline Blood Chemical Test & $\checkmark$ & & & & & & & $\checkmark$ \\
\hline $\begin{array}{c}\text { Urine microalbumin, } \\
\text { creatinine }\end{array}$ & $\checkmark$ & & & & & & & $\checkmark$ \\
\hline$E C G$ & $\checkmark$ & & & & & & & $\checkmark$ \\
\hline $\begin{array}{c}\text { Arterial stiffness } \\
(A B I, P W V)\end{array}$ & $\checkmark$ & & & & & & & $\checkmark$ \\
\hline Records for Diet & & & $\checkmark$ & $\checkmark$ & $\checkmark$ & $\checkmark$ & $\checkmark$ & $\checkmark$ \\
\hline Patient Compliance & & & $\checkmark$ & $\checkmark$ & $\checkmark$ & & & \\
\hline Adverse Events & & & $\checkmark$ & $\checkmark$ & $\checkmark$ & $\checkmark$ & $\checkmark$ & $\checkmark$ \\
\hline $\begin{array}{r}\text { Combined Medications } \\
\text { for Accompanied } \\
\text { Diseases }\end{array}$ & & & $\checkmark$ & $\checkmark$ & $\checkmark$ & $\checkmark$ & $\sqrt{ }$ & $\sqrt{ }$ \\
\hline fecal sample collection & $\checkmark$ & & & $\checkmark$ & $\checkmark$ & $\checkmark$ & $\checkmark$ & $\checkmark$ \\
\hline
\end{tabular}




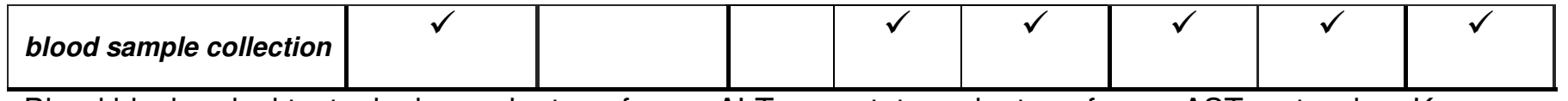

Blood biochemical test: alanine aminotransferase ALT, aspartate aminotransferase AST, potassium K, sodium $\mathrm{Na}$, chloride $\mathrm{Cl}$, urea nitrogen BUN, creatinine CREA, uric acid UA, total cholesterol TC, triglyceride TG, high density lipoprotein cholesterol HDL-C, low density lipoprotein cholesterol LDL-C, fasting blood glucose GLU.

\section{Sample size $\{14\}$}

A sample size calculation was performed via PASS Version 15 software before trial initiation based on followings: 1) no relevant data up-to-date for FMT on hypertension; 2) a clinically significant improvement of a decrease in office SBP at least $5 \mathrm{mmHg}$ associated with reduction in stroke(14\%), cardiovascular disease (9\%) and mortality (7\%) (33); 3) meta-analysis of effectiveness of probiotics on hypertension based on randomized controlled clinical trials revealed a reduction of SBP by $3.56 \mathrm{mmHg}(95 \% \mathrm{Cl}, 6.46$ to 0.66$)$ (34). Assuming a true SBP difference between "FMT capsule group" and "placebo group" with a means of $5 \mathrm{mmHg}$ and a standard deviation of $8.6 \mathrm{mmHg}$ for SBP change per arm, a sample size of 96 grade 1 hypertensive patients yields $>80 \%$ statistical power to demonstrate a $>5 \mathrm{mmHg}$ difference between arms at a 1-sided alpha level 0.025 . Give premature withdraw or failure potentials before primary end point collected, a $20 \%$ rate of loss-of-follow up is calculated with 120 participants with grade 1 hypertension according to the "2010 Chinese Guidelines for Prevention and Treatment of Hypertension" (29) recruited and randomized 1:1 to receive FMT by capsules or placebo capsules.

\section{Recruitment $\{15\}$}

Eligible participants will be screened at hypertension outpatient clinic of the implementation centers, according to eligible criteria for the trial. Advertisements for study utilizing onsite posters and multiple media platforms are promoted.

\section{Assignment of interventions: allocation}

\section{Sequence generation $\{16 \mathrm{a}\}$}

Randomization allocation sequencing is performed by an independent statistician via SAS 9.4 software(SAS Institute, Cary NC, USA) and incorporated into a central computerized randomization system which is developed and managed by the Medical Research \& Biometrics Center, National Center for Cardiovascular Diseases, Fuwai Hospital Chinese Academy of Medical Sciences.

\section{Concealment mechanism $\{16 b\}$}

Only the staff from the Medical Research \& Biometrics Center who generate the random codes and an independent staff from the responsible center (Fuwai Hospital) reserve the overall random codes which are incorporated into the Electronic Data Capture System(EDC system) and also reserved with sealed envelopes for each participant.

\section{Implementation $\{16 c\}$}

Patient care providers and investigators from implementation centers will log in to the EDC system, enter the system login page, select the center and fill in the password, and then enter the randomization information page. After filling the name of implementation center, qualified screening code, initials of participants' name, age, sex, and other relevant information, passing the system check for information correction, and clicking the "submit for confirmation" button, the randomization 
date, qualified random number and allocated arm of participants will be obtained. At this time, participants are enrolled in this study.

The eligible participants will be randomly divided into two arms, in which the "FMT capsule" group and "Placebo capsule" group will receive FMT capsules or placebo capsules in separate for three times in the outpatient at Day 1, Day 7 and Day 14 after trial initiation. The random numbers are labelled on package for capsules in accordance with GCP. The first administration (Day 1) will be commenced after randomization with medication compliance/adherence, adverse events, and treatment for comorbid diseases assessed and recorded via investigators and/or patient care providers.

\section{Assignment of interventions: Blinding}

\section{Who will be blinded $\{17 \mathrm{a}\}$}

Study participants and all personnel including investigators and/or patient care providers who recruit and follow-up participants and collect data are blinded. Packaged intervention capsules and sealed emergency envelopes for each participant are prepared by independent staff from the responsible center not involving in the study. The sealed envelopes are reserved at the responsible center in case for disclosure of arm allocation when necessary.

\section{Procedure for unblinding if needed $\{17 \mathrm{~b}\}$}

If medical emergency events occur, unblinding can be performed if necessary, via the responsible investigation team under the supervision of principal investigator. The reason, date, location of unblinding and signature from investigators will be recorded in detail. All participants will be followed up until close-out visit unless the withdrawal criteria are met.

\section{Data collection and management}

\section{Plans for assessment and collection of outcomes $\{18 \mathrm{a}\}$}

All outcomes are evaluated at seven timepoints, including baseline, the first intervention, and 7 days, 14 days, 30 days, 60 days, and 90 days onwards. In addition to kicking-off meeting on February 26,2021, all staff involving the study has been trained for detailed study protocol, participant recruitment and informed consent, clinical assessment, sample collection and storage, data collection utilizing printed CRFs and EDC system , and etc.

\section{The week prior to enrollment (-1 0 week)}

The following procedures will be finished:

1) Obtaining written informed consent from participants for the study and completing the screening questionnaire.

2) Recording office blood pressure and heart rate.

3) Recording previous history, current- and prior-treatment, and other documented eligibility factors.

4) Recording personal information such as height, weight, and waist circumference.

5) Examinations including 24-hour ABPM, electrocardiography, blood routine, blood biochemical test, urine routine, urine microalbumin, creatinine and arterial stiffness assessment (PWV, $A B I)$.

6) Reconfirmation for participant eligibility according to the inclusion and exclusion criteria.

7) Fulfilling baseline case report form (CRF) with printed CRF and EDC system.

8) Collecting fecal and blood samples. 
9) Appointment for randomization visit (within a week after screening).

\section{Randomization (Day 0)}

Participants will be randomly assigned to receive the first capsule treatment (Day 1) with dietary history collected before treatment.

\section{Treatment Period (Day 1, Day 7, Day 14)}

The eligible subjects will be randomly divided into two arms, in which the "FMT capsule" group and "Placebo capsule" group will receive FMT via capsules or placebo capsules in separate for three times in the outpatient clinics at Day 1, Day 7 and Day 14 after trial initiation. At each visit, medication adherence, adverse events, treatment for comorbid diseases, and dietary history will also be assessed and recorded.

\section{Follow-up Visits (Day 7, Day 14, Day 30, Day 60)}

1) Recording office blood pressure and heart rate, and on Day 30 visit performing 24-h ABPM;

2) Recording any reasons for discontinuing intervention;

3) Recording adverse events, participant adherence to treatment regimens, and changes in combined medication for comorbid diseases, and non-study therapy;

4) Recording dietary history;

5) Collecting blood and fecal samples;

6) Fulfilling the follow-up CRFs with printed CRF and EDC system;

7) Appointment for the next follow-up visit.

\section{Final Visit (Day 90)}

Participants will undergo a comprehensive examination to evaluate the efficacy of FMT on hypertension, including: physical examination, blood pressure, heart rate, 24-h ABPM, blood routine, urine routine, blood biochemical test, urine microalbuminuria protein, creatinine, electrocardiography, and arterial stiffness assessment (ABI, PWV). In addition, dietary history, adverse events and combined medications for comorbid disease will be recorded, and all CRFs will be reviewed. As well, effective colonization and durability of intestinal flora in hypertensive recipients after oral administration of "FMT capsules" or "placebo capsules", and the underlying mechanism of $\mathrm{BP}$ change and fecal microbiota, will be analyzed via metagenomic and metabolomic profiling analysis.

\section{Plans to promote participant retention and complete follow-up $\{18 b\}$}

All enrolled participants will receive a comprehensive health evaluation via a professional physician, and will take FMT capsules or placebo capsules for free. In the average follow-up of three months, participants will receive consultation from professionals, medication guidance, and follow-up services for free. In order to assist on travel costs and other expenses for take part in the study, subsidies will be provided for each participant per visit. In terms of participants refusing to follow the allocated intervention algorithm or occurring with adverse events, follow-up in reference to evaluation plan will continue. The reason for withdrawal (and degree of withdrawal) will be record for all participants who withdraw from the study or who are not thorough followed up. Although withdrawal, clinical treatment and other rights and interests for patients will not be affected.

\section{Data management $\{19\}$}

Data collection will be performed via the printed CRF and then entered into Electronic Data Capture (EDC) system( $h$ ttp://47.107.145.115/fmtEDC/) developed by the National Center for Cardiovascular Diseases, enabling repeatedly confirmation from the patient care providers and/or investigators from the implementation centers. All relative staff will be trained and e-mailed with account and password for using the EDC system with signed meeting summaries. The EDC system will automatically check the data format, rules and range, which will then be rechecked manually. All printed CRF and examination results will be scanned and sent to the Collaborating Office with two staff independently reconfirm the accuracy of the data. Changes for data are allowed and tracked with reasons in the EDC system. Also, a third party is invited to supervise data quality. After completion of the study, the 
EDC database will turn to lock state with the blinding audit report generated by the statistician and confirmed by the principal investigator, responsible data manager and statistical analyst. Data will be reserved for five years after study completion.

\section{Confidentiality $\{27\}$}

In terms of confidentiality protection for participants, personal information of participants including name, address, contact, personal ID numbers will be kept within investigation centers and prohibited for any thirty party. In case of data collection (i.e. metagenomic profiling, metabolomic profiling, clinical information and examination results), all personal identifiers will be removed with alternatives such as unique screening code and randomization code used.

\section{Plans for collection, laboratory evaluation and storage of biological specimens for genetic or molecular analysis in this trial/future use $\{33\}$}

Except for routine clinical laboratory test for blood and urine samples (will not be stored) as above description, study blood $(10 \mathrm{ml})$ and fecal samples $(5 \mathrm{~g})$ will be collected at baseline and at Day $7( \pm$ 1 day), Day 14 ( \pm 1 day), Day 30( \pm 3 days), Day 60 ( \pm 3 days) and Day 90 ( \pm 3 days). Blood samples will be centrifuged immediately with supernatants and blood cells collected in separate. These samples will be stored at $-80^{\circ} \mathrm{C}$ freezers and delivered to the responsible center via dry ice for further metagenomic and metabolomic profiling analysis. Other experiments such as specific bacteria isolation from fecal samples may be developed for addressing the scientific question of the study.

\section{Statistical methods}

\section{Statistical methods for primary and secondary outcomes $\{20 \mathrm{a}\}$}

All primary analysis for end points will be based on the intent-to-treatment principal including all randomized patients, regardless of adherence. For primary endpoint analysis, analysis of covariance adjusting for baseline SBP and centers will be performed. In terms of baseline data and secondary outcomes, categorical variables will be described as frequency (percentage, \%) with differences between arms evaluated through Cohran-Mantel-Haensel chi-square test or Fisher's exact test as appropriate with center effect adjustment. Continuous variables were presented as mean (standard deviation, SD), or median (interquartile range, IQR) with t-test or Wilcoxon test performed as appropriate. Assessment of interventions on the change of continuous data will perform in the mixedeffect models or the analysis of covariance. All statistical tests for clinical metadata will be employed via SAS ${ }^{89} 9.4$ software or R software (Version 4.0.2). A significant level of one-sided 0.025 will be utilized for primary endpoint analysis, and significant $p$ value for other statistical analyses is defined as a level of 2 -sided 0.05 .

\section{Interim analyses $\{21 b\}$}

The interim analysis is deemed unnecessary for this study, given follow-up conditions: 1) short-term study period with only three-month follow-up for each participant and risk of type I error of interim analysis; 2) a relatively small sample size of 120 patients and double-blinded setting of study design.

Methods for additional analyses (e.g. subgroup analyses) $\{20 \mathrm{~b}\}$ 
Further sensitivity analysis for outcome consistency will be evaluated in multiple subgroups such as age, sex, baseline Office SBP level, implementation sites, history of diabetes or hyperlipidemia at randomization, proportion of donor microbiota engraftment, etc.

\section{Methods in analysis to handle protocol non-adherence and any statistical methods to handle missing data $\{20 \mathrm{c}\}$}

Per-protocol analysis will also be performed for randomized patients successfully treated excluding those presenting with major deviations (i.e. violation of core inclusion and/or exclusion criteria, use of forbidden drugs, intervention protocol violation, etc.). In terms of missing data handling, the multiple imputation method as primary analysis will be used for primary endpoint. Additional sensitivity analysis will be performed for dataset using last-observation-carried-forward(LOCF) method and for complete dataset excluding missing data. For secondary endpoints, missing data will not be handled because of exploratory analysis.

\section{Plans to give access to the full protocol, participant level-data and statistical code} $\{31 \mathrm{c}\}$

The final protocol for study and statistical analysis will be identified before lock of the EDC database and updated onto the ClinicalTrials website. As well, the full protocol, de-identified participant-level dataset and statistical code will be shared along with publication according to the journal requirement.

\section{Oversight and monitoring}

\section{Composition of the coordinating centre and trial steering committee $\{5 \mathrm{~d}\}$}

The overall study is coordinated and oversighted by the Collaborating Office and the Quality Control Committee following the Good Clinical Practice(GCP) principles with online catch-up meeting every week. As well, the web-based EDC system allow for real-time monitoring and recheck with scanned CRFs and examination reports via staffs. Each implementation center will also organize a local investigation team including the local principal investigator ensuring study protocol implementation and quality control, trained investigators and/or patient care providers for participant screening, recruitment, follow-up and adverse event handling, staffs for data collection and storage, specimen collection, processing and storage.

\section{Composition of the data monitoring committee, its role and reporting structure $\{21 \mathrm{a}\}$}

Staffs of Collaborating Office and a third party from Medical Research \& Biometrics Center, National Center for Cardiovascular Diseases, Fuwai Hospital Chinese Academy of Medical Sciences has been invited for data monitoring with periodical report to the data and safety monitoring committee, among which all personnel completely independent from the funders and any competing interests.

\section{Adverse event reporting and harms $\{22\}$}

Potential risks for FMT include transmission of currently unknown infectious agents or diseases related to donor gut microbiota, although profound screening process performed according to current consensus. Other previously reported adverse events are often self-limiting such as diarrhea and 
nausea, particularly for transplantation via oral capsules (27). Since the first intervention, all adverse events will be recorded on the AE page of CRF and EDC system with details of onset date and time, severity, procedures, correlation and influence on study interventions, prognosis and date. The severity of AEs and their correlation with study interventions will be assessed according to the Common Terminology Criteria for Adverse Events (CTCAE Version 4.03, http://evs.nci.nih.gov/ftp1/CTCAE/CTCAE_4.03_2010-06-14_QuickReference_8.5x11.pdf.) with a five-grading scale presented. Severe adverse events (SAEs) are defined as events leading to mortality, needs for or prolonged hospitalization, persistent or severe morbidity, and/or medical emergency determined by investigators in accordance with GCP. Investigators will deal with and report SAEs to the local ethics committee immediately and to the responsible center and principal investigator within 24 hours.

\section{Frequency and plans for auditing trial conduct $\{23\}$}

Conduct of the study will be periodically audited online and onsite by qualified staffs of Collaborating Office and the Quality Control Committee, with experiences from the Strategy of Blood Pressure Intervention in Elderly Hypertensive Patients (STEP) trial (35).

\section{Plans for communicating important protocol amendments to relevant parties (e.g. trial participants, ethical committees) $\{25\}$}

Study protocol will not be changed without the written approval from the Ethics Committee of the responsible center. Protocol amendments will also be submitted to the ethics committee of implementation centers, investigators and participants.

\section{Dissemination plans $\{31 a\}$}

All unpublished information and data related to the study are strictly confidential and will be utilized only for this study, agreed by all investigators. The study results will be shared with the participants, published in peer-reviewed journals, and/or presented on academic conferences. De-identified data will be updated on secure online database such as European Nucleotide Archive (ENA, https://www.ebi.ac.uk/ena/submit/sra/) along with publication requirement from the journal. Access to these de-identified data will be required for written permission from the responsible investigation center and only for qualified researchers.

\section{Discussion}

A multitude of evidence indicate non-communicable diseases from microbe-free involvement to association or even cause-and-effect relationships with microbial dysbiosis, in the context of different environmental and genetic predisposition(36). Recent patient- and animal model-based studies have yield distinct gut microbiota composition and function accompanying with host hypertensive or prehypertensive state $(5,7-10)$. Of note is our previous study revealed transfer of elevated BP phenotype from hypertensive patients to GF rodents, which confirmed a cause-and-effect relationship between gut microbiota and BP elevation(9). A hypothesis of intervention on gut microbiota was thus proposed with multiple vehicles under investigation up-to-date such as prebiotics, probiotics, and antibiotics. Prebiotics are dietary components (i.e. fermentable fibers) interfering with microbes and probiotics are living beneficial microbes, in some cases contributing to improved gut barrier function and host homeostasis (37). Recent study proved that high-fiber diet and acetate supplement showed significant BP-lowering and heart-/kidney-protecting effects in DOCA-salt hypertensive models (38). Also, probiotics such as bacteria belongs to Lactobacillus $(39,40)$ presented BP-lowering effect on 
both animals models and human trials, with a potential mechanism explanation of angiotensinconverting enzyme inhibition (41-43). However, meta-analysis of current prebiotics (15) or probiotics (34) generally presented modest BP-lowering effect with $-0.9 \mathrm{mmHg}(95 \% \mathrm{Cl}-2.5$ to $-0.6 \mathrm{mmHg})$ and $-3.56 \mathrm{mmHg}(95 \% \mathrm{Cl}-6.46$ to $-0.66 \mathrm{mmHg})$ for SBP reduction, respectively. The latter metaanalysis also revealed a threshold of duration(>8 weeks) and daily dose(probiotics $>1011$ colonyforming units), and a recommendation on multiple bacteria prescribed for effective intervention(34) . As well, innovative products such as mixed strains of live bacteria belongs to Lactobacilus and Bifidobacteria are still under trial (44). Limited evidences on specific microbes causing hypertension prohibit explorations on microorganism-targeting supplement via prebiotics and/or probiotics, or inhibit via antibiotics. In addition, board spectrum antibiotics, which are commonly utilized for infectious disease treatment and pre-intervention of experimental models for microbiome studies, probably disrupt microbial homeostasis with potential loss of beneficial species or strains, and cumulatively increasing future risk of antibiotic-resistant microbes and even of pathogenic conditions such as obesity, diabetes, asthma and inflammatory bowel diseases (45-49). Our study thus focused on a methodology of entire healthy gut microbiota restoration(FMT) and already performed or investigated in multiple infectious diseases and non-communicable diseases. In addition, oral capsules are used to reduce the risk for invasive delivery methods such as upper endoscopy, colonoscopy, and nasoenteric tubes(27). This study is part of the clinical translational section of our research project "The Role of Gut Microbiota in the Pathogenesis of Essential Hypertension" (Project ID 81630014, funded by National Natural Science Foundation of China). The study firstly in the world explores the effect of FMT on hypertension with cardiovascular event-associated indicator(33), office SBP, as primary outcome, and other BP-related indicators including office DBP, BP indicators from ABPM and home BP monitoring as main secondary endpoints. Target population is constrained to patients with grade 1 hypertension identified from the "2010 Chinese guidelines for the management of hypertension" (29), in whom non-pharmacological treatment options can be recommended first for those without secondary causes, severe target organ damage or accompanied diseases. Further explorations on changes of recipient microbiome and metabolome via multi-omics approaches are planned for advanced understanding the role of specific microbes in hypertension. In terms of stability for microbiota, products interfering with microbiota such as antibiotics or probiotics are prohibited along with the 3-month study period. Given the recalling bias and practical condition for challenging recruitment, duration of a month for antibiotics or probiotics use prior to recruitment is involved in exclusion criteria. Also, a strict multi-center, centralized randomization, double-blinded, placebo-controlled trial setting is performed to ensure the reliability of study results.

In summary, this is the first study aims to provide high-quality evidence for microbiota intervention on treating hypertension via fecal microbiota transplantation, as advancement and clinical translation of our previous research on the role of gut microbiota in the pathogenesis of hypertension. Further investigations on specific microorganism or relative postbiotics may also be developed from this study via multiple approaches such as metagenomic sequencing and metabolomic profiling.

\section{Trial status}

This article is in accordance with study protocol (Version 3.3, dated 26 February 2021). Participant recruitment started on 17 March 2021 with 63 participants recruited in current and is anticipated to reach December 2021.

\section{Abbreviations}

FMT: fecal microbiota transplantation; CVD: cardiovascular disease; BP: blood pressure; GF: germ free; IBD: inflammatory bowel disease; BMI: body mass index; ABI: ankle branchial index; SBP: systolic blood pressure; DBP: diastolic blood pressure; LAACI: large artery atherosclerotic cerebral 
infarction; TIA: transient ischemic attack; $\mathrm{PCI}$ : percutaneous transluminal coronary intervention; CABG: coronary artery bypass grafting; NYHA: New York Heart Association; ABPM: 24-hour ambulatory blood pressure monitoring; AE: adverse event; ECG: electronic cardiography; PWV: pulse wave velocity; ALT: alanine aminotransferase; AST: aspartate aminotransferase; K: potassium; Na: sodium; Cl: chloride; BUN: urea nitrogen; CREA: creatinine; UA: uric acid; TC: total cholesterol; TG: triglyceride; HDL-C: high density lipoprotein cholesterol; LDL-C: low density lipoprotein cholesterol; GLU: fasting blood glucose; EDC: Electronic Data Capture; GCP: Good Clinical Practice; ID, identification; CRF: case report form; SD, standard deviation; IQR, interquartile range; LOCF: last-observation-carried-forward; CTCAE: Common Terminology Criteria for Adverse Events; STEP: Strategy of Blood Pressure Intervention in Elderly Hypertensive Patients; ENA: European Nucleotide Archive

\section{Declarations}

\section{Acknowledgements}

We thank Wei Li, Yang Wang, Yi Sun, Qianqian Wei from Medical Research \& Biometrics Center, National Center for Cardiovascular Diseases, Fuwai Hospital Chinese Academy of Medical Sciences for statistical analysis consultation, EDC system and centralized randomization system construction, and data management. We appreciate all personnel involving in the study from the investigation centers and collaborating parties including but not limited to Xiurong Guo, Lu Wang, Hailei Wu, Shutong Wang, Lu Cheng, Xuefang Yan, Zhen Chen, Dan Qi, Changyi Zhang, Weiqiang Wu, Zhichao Chen, Yonghong Yuan, Ruishan Zhang, Xian Qiu, Yun Ti, Yajing Zhao, Wen Zhang, Youqing Liang, Yunjian Li, Qian Wang, and all study participants for their contribution.

\section{Authors' contributions $\{31 \mathrm{~b}\}$}

$\mathrm{JC}$ is the chief investigator who conceived and is responsible for the study, and procured funding. LF and JC designed the study and drafted the original protocol. LF, YZ and YW operated statistical planning and calculated the sample size. YW generated the random codes. LW, JR, YC, PB, ZG, WM, BZ, and JY contributed to study design and to collect the data. All authors have read and approved the final protocol.

\section{Funding $\{4\}$}

This work is supported by the National Natural Science Foundation of China (Project ID. 81630014) and Beijing Outstanding Young Scientist Program (Project ID, BJJWZYJH01201910023029), Beijing Municipal Education Commission.

\section{Availability of data and materials $\{29\}$}

The investigators have access to the final trial dataset. The study funders will not involve the study 
design, implementation, data interpretation, and result publication.

\section{Ethics approval and consent to participate $\{24\}$}

This study is approved by the Ethics Committee of Fuwai Hospital, Chinese Academy of Medical Sciences (approval NO.2020-1334, Date 14 $4^{\text {th }}$ April, 2020) and the Ethics Committee of all collaborating implementation centers. Written, informed consents before participant enrollment are required.

\section{Consent for publication $\{32\}$}

Not applicable.

\section{Competing interests $\{28\}$}

The authors declare that they have no competing interests.

\section{Authors' information (optional)}

1. Hypertension Center, State Key Laboratory of Cardiovascular Disease, National Center for Cardiovascular Diseases, Fuwai Hospital, Chinese Academy of Medical Sciences and Peking Union Medical College, Beijing, China.

2. Shanxi Bethune Hospital, Taiyuan, Shanxi, China

3. The Second Affiliated Hospital of Shantou University, Shantou, Guangdong, China

4. Medical Research \& Biometrics Center, National Center for Cardiovascular Diseases, Fuwai Hospital Chinese Academy of Medical Sciences, Beijing, China

5. Fuwai Yunnan Cardiovascular Hospital, Kunming, Yunnan, China

6. Qilu Hospital of Shandong University, Jinan, Shandong, China

7. The People's Hospital of Ji Xian District, Tianjin, China

8. Southern University of Science and Technology Hospital, Shenzhen, China

\section{References}

1. Roth GA, Johnson C, Abajobir A, Abd-Allah F, Abera SF, Abyu G, et al. Global, Regional, and National Burden of Cardiovascular Diseases for 10 Causes, 1990 to 2015. J Am Coll Cardiol. 2017;70(1):1-25.

2. Roth GA, Nguyen G, Forouzanfar MH, Mokdad AH, Naghavi M, Murray CJ. Estimates of global and regional premature cardiovascular mortality in 2025. Circulation. 2015;132(13):1270-82.

3. Global, regional, and national comparative risk assessment of 79 behavioural, environmental and occupational, and metabolic risks or clusters of risks, 1990-2015: a systematic analysis for the Global Burden of Disease Study 2015. Lancet. 2016;388(10053):1659-724.

4. Organization WH. Noncommunicable diseases country profiles 2018. 2018.

5. Muralitharan RR, Jama HA, Xie L, Peh A, Snelson M, Marques FZ. Microbial Peer Pressure:

The Role of the Gut Microbiota in Hypertension and Its Complications. Hypertension.

2020;76(6):1674-87. 
6. Karbach SH, Schönfelder T, Brandão I, Wilms E, Hörmann N, Jäckel S, et al. Gut Microbiota Promote Angiotensin II-Induced Arterial Hypertension and Vascular Dysfunction. J Am Heart Assoc. 2016;5(9).

7. Sun S, Lulla A, Sioda M, Winglee K, Wu MC, Jacobs DR, Jr., et al. Gut Microbiota Composition and Blood Pressure. Hypertension. 2019;73(5):998-1006.

8. Yang T, Santisteban MM, Rodriguez V, Li E, Ahmari N, Carvajal JM, et al. Gut dysbiosis is linked to hypertension. Hypertension. 2015;65(6):1331-40.

9. Li J, Zhao F, Wang Y, Chen J, Tao J, Tian G, et al. Gut microbiota dysbiosis contributes to the development of hypertension. Microbiome. 2017;5(1):14.

10. Kim S, Goel R, Kumar A, Qi Y, Lobaton G, Hosaka K, et al. Imbalance of gut microbiome and intestinal epithelial barrier dysfunction in patients with high blood pressure. Clin Sci (Lond). 2018;132(6):701-18.

11. Adnan S, Nelson JW, Ajami NJ, Venna VR, Petrosino JF, Bryan RM, Jr., et al. Alterations in the gut microbiota can elicit hypertension in rats. Physiol Genomics. 2017;49(2):96-104.

12. Yan X, Jin J, Su X, Yin X, Gao J, Wang X, et al. Intestinal Flora Modulates Blood Pressure by Regulating the Synthesis of Intestinal-Derived Corticosterone in High Salt-Induced Hypertension. Circ Res. 2020;126(7):839-53.

13. Galla S, Chakraborty S, Cheng X, Yeo J, Mell B, Zhang H, et al. Disparate effects of antibiotics on hypertension. Physiol Genomics. 2018;50(10):837-45.

14. Qi D, Nie XL, Zhang JJ. The effect of probiotics supplementation on blood pressure: a systemic review and meta-analysis. Lipids Health Dis. 2020;19(1):79.

15. Evans CE, Greenwood DC, Threapleton DE, Cleghorn CL, Nykjaer C, Woodhead CE, et al. Effects of dietary fibre type on blood pressure: a systematic review and meta-analysis of randomized controlled trials of healthy individuals. J Hypertens. 2015;33(5):897-911.

16. Dong JY, Szeto IM, Makinen K, Gao Q, Wang J, Qin LQ, et al. Effect of probiotic fermented milk on blood pressure: a meta-analysis of randomised controlled trials. Br J Nutr. 2013;110(7):1188-94.

17. Wilck N, Matus MG, Kearney SM, Olesen SW, Forslund K, Bartolomaeus H, et al. Saltresponsive gut commensal modulates $T(H) 17$ axis and disease. Nature. 2017;551(7682):585-9.

18. Surawicz CM, Brandt LJ, Binion DG, Ananthakrishnan AN, Curry SR, Gilligan PH, et al. Guidelines for diagnosis, treatment, and prevention of Clostridium difficile infections. Am J Gastroenterol. 2013;108(4):478-98.

19. Cammarota G, Ianiro G, Tilg $H$, Rajilić-Stojanović M, Kump P, Satokari R, et al. European consensus conference on faecal microbiota transplantation in clinical practice. Gut. 2017;66(4):569-80.

20. Wang H, Cui B, Li Q, Ding X, Li P, Zhang T, et al. The Safety of Fecal Microbiota Transplantation for Crohn's Disease: Findings from A Long-Term Study. Adv Ther. 
2018;35(11):1935-44.

21. Costello SP, Hughes PA, Waters O, Bryant RV, Vincent AD, Blatchford P, et al. Effect of Fecal Microbiota Transplantation on 8-Week Remission in Patients With Ulcerative Colitis: A

Randomized Clinical Trial. Jama. 2019;321(2):156-64.

22. Halkjær SI, Christensen AH, Lo BZS, Browne PD, Günther S, Hansen LH, et al. Faecal microbiota transplantation alters gut microbiota in patients with irritable bowel syndrome: results from a randomised, double-blind placebo-controlled study. Gut. 2018;67(12):2107-15.

23. Li SS, Zhu A, Benes V, Costea PI, Hercog R, Hildebrand F, et al. Durable coexistence of donor and recipient strains after fecal microbiota transplantation. Science. 2016;352(6285):586-9.

24. Kootte RS, Levin E, Salojärvi J, Smits LP, Hartstra AV, Udayappan SD, et al. Improvement of Insulin Sensitivity after Lean Donor Feces in Metabolic Syndrome Is Driven by Baseline Intestinal Microbiota Composition. Cell Metab. 2017;26(4):611-9.

25. Yu EW, Gao L, Stastka P, Cheney MC, Mahabamunuge J, Torres Soto M, et al. Fecal microbiota transplantation for the improvement of metabolism in obesity: The FMT-TRIM doubleblind placebo-controlled pilot trial. PLoS Med. 2020;17(3):e1003051.

26. Ng SC, Xu Z, Mak JWY, Yang K, Liu Q, Zuo T, et al. Microbiota engraftment after faecal microbiota transplantation in obese subjects with type 2 diabetes: a 24-week, double-blind, randomised controlled trial. Gut. 2021._doi: 10.1136/gutjnl-2020-323617.

27. Allegretti JR, Mullish BH, Kelly C, Fischer M. The evolution of the use of faecal microbiota transplantation and emerging therapeutic indications. Lancet. 2019;394(10196):420-31.

28. Chan AW, Tetzlaff JM, Gøtzsche PC, Altman DG, Mann H, Berlin JA, et al. SPIRIT 2013 explanation and elaboration: guidance for protocols of clinical trials. Bmj. 2013;346:e7586. 29. Liu LS. 2010 Chinese guidelines for the management of hypertension. Zhonghua Xin Xue Guan Bing Za Zhi. 2011;39(7):579-615.

30. Haifer C, Kelly CR, Paramsothy S, Andresen D, Papanicolas LE, McKew GL, et al. Australian consensus statements for the regulation, production and use of faecal microbiota transplantation in clinical practice. Gut. 2020;69(5):801-10.

31. Sokol H, Galperine T, Kapel N, Bourlioux P, Seksik P, Barbut F, et al. Faecal microbiota transplantation in recurrent Clostridium difficile infection: Recommendations from the French Group of Faecal microbiota Transplantation. Dig Liver Dis. 2016;48(3):242-7.

32. Ng SC, Kamm MA, Yeoh YK, Chan PKS, Zuo T, Tang W, et al. Scientific frontiers in faecal microbiota transplantation: joint document of Asia-Pacific Association of Gastroenterology (APAGE) and Asia-Pacific Society for Digestive Endoscopy (APSDE). Gut. 2020;69(1):83-91. 33. Whelton PK, He J, Appel LJ, Cutler JA, Havas S, Kotchen TA, et al. Primary prevention of hypertension: clinical and public health advisory from The National High Blood Pressure Education Program. Jama. 2002;288(15):1882-8.

34. Khalesi S, Sun J, Buys N, Jayasinghe R. Effect of probiotics on blood pressure: a systematic 
review and meta-analysis of randomized, controlled trials. Hypertension. 2014;64(4):897-903. 35. Zhang S, Wu S, Ren J, Chen X, Zhang X, Feng Y, et al. Strategy of blood pressure intervention in the elderly hypertensive patients (STEP): Rational, design, and baseline characteristics for the main trial. Contemp Clin Trials. 2020;89:105913.

36. Finlay $\mathrm{BB} \mathrm{CH}$, the Microbiome. Are noncommunicable diseases communicable? Science. 2020;367(6475):250-1.

37. Gibson GR, Hutkins R, Sanders ME, Prescott SL, Reimer RA, Salminen SJ, et al. Expert consensus document: The International Scientific Association for Probiotics and Prebiotics (ISAPP) consensus statement on the definition and scope of prebiotics. Nat Rev Gastroenterol Hepatol. 2017;14(8):491-502.

38. Marques FZ, Nelson E, Chu PY, Horlock D, Fiedler A, Ziemann M, et al. High-Fiber Diet and Acetate Supplementation Change the Gut Microbiota and Prevent the Development of Hypertension and Heart Failure in Hypertensive Mice. Circulation. 2017;135(10):964-77.

39. Gareau MG, Sherman PM, Walker WA. Probiotics and the gut microbiota in intestinal health and disease. Nat Rev Gastroenterol Hepatol. 2010;7(9):503-14.

40. Lewis-Mikhael AM, Davoodvandi A, Jafarnejad S. Effect of Lactobacillusplantarum containing probiotics on blood pressure: A systematic review and meta-analysis. Pharmacol Res. 2020;153:104663.

41. Yang G, Jiang Y, Yang W, Du F, Yao Y, Shi C, et al. Effective treatment of hypertension by recombinant Lactobacillus plantarum expressing angiotensin converting enzyme inhibitory peptide. Microb Cell Fact. 2015;14:202.

42. Carter CS, Morgan D, Verma A, Lobaton G, Aquino V, Sumners E, et al. Therapeutic Delivery of Ang(1-7) via Genetically Modified Probiotic: A Dosing Study. J Gerontol A Biol Sci Med Sci. 2020;75(7):1299-303.

43. Nakamura Y, Yamamoto N, Sakai K, Takano T. Antihypertensive effect of sour milk and peptides isolated from it that are inhibitors to angiotensin I-converting enzyme. J Dairy Sci. 1995;78(6):1253-7.

44. Mähler A, Wilck N, Rauch G, Dechend R, Müller DN. Effect of a probiotic on blood pressure in grade 1 hypertension (HYPRO): protocol of a randomized controlled study. Trials. 2020;21(1):1032.

45. Bailey LC, Forrest CB, Zhang P, Richards TM, Livshits A, DeRusso PA. Association of antibiotics in infancy with early childhood obesity. JAMA Pediatr. 2014;168(11):1063-9.

46. Mikkelsen KH, Knop FK, Frost M, Hallas J, Pottegård A. Use of Antibiotics and Risk of Type 2 Diabetes: A Population-Based Case-Control Study. J Clin Endocrinol Metab. 2015;100(10):363340.

47. Blaser MJ. Antibiotic use and its consequences for the normal microbiome. Science. 2016;352(6285):544-5. 
48. Hoskin-Parr L, Teyhan A, Blocker A, Henderson AJ. Antibiotic exposure in the first two years of life and development of asthma and other allergic diseases by $7.5 \mathrm{yr}$ : a dose-dependent relationship. Pediatr Allergy Immunol. 2013;24(8):762-71.

49. Kronman MP, Zaoutis TE, Haynes K, Feng R, Coffin SE. Antibiotic exposure and IBD development among children: a population-based cohort study. Pediatrics. 2012;130(4):e794-803. 


\section{Supplementary Files}

This is a list of supplementary files associated with this preprint. Click to download.

- Ethicsdocument.pdf

- Originalfundingdocument.pdf 